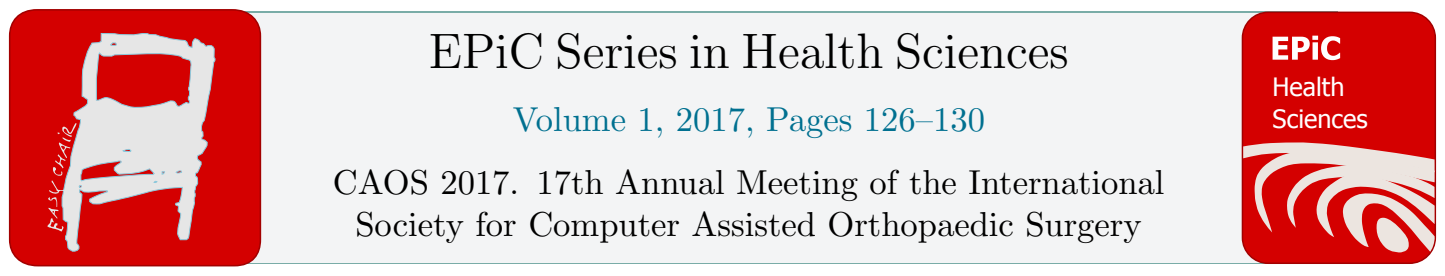

\title{
Robot-Bone Attachment Device for Robot- Assisted Percutaneous Bone Fragment Manipulation
}

\author{
Giulio Dagnino ${ }^{1}$, Ioannis Georgilas ${ }^{2}$, Kostas Georgilas ${ }^{3}$, Paul Köhler ${ }^{1}$, \\ Samir Morad ${ }^{4}$, Peter Gibbons ${ }^{1}$, Roger Atkins ${ }^{5}$, and Sanja Dogramadzi ${ }^{1}$ \\ ${ }^{1}$ Bristol Robotics Laboratory - UWE, Bristol, U.K. \\ ${ }^{2}$ University of Bath, Bath, U.K. (was with ${ }^{1}$ ) \\ ${ }^{3}$ University of Aberdeen, Aberdeen, U.K. \\ ${ }^{4}$ Aston University, Birmingham, U.K. (was with ${ }^{1}$ ) \\ ${ }^{5}$ University Hospitals, Bristol, U.K. \\ giulio.dagnino@brl.ac.ukm, sanja.dogramadzi@uwe.ac.uk
}

\begin{abstract}
The treatment of joint-fractures is a common task in orthopaedic surgery causing considerable health costs and patient disabilities. Percutaneous techniques have been developed to mitigate the problems related to open surgery (e.g. soft tissue damage), although their application to joint-fractures is limited by the sub-optimal intra-operative imaging (2D-fluoroscopy) and by the high forces involved. Our earlier research toward improving percutaneous reduction of intra-articular fractures has resulted in the creation of a robotic system prototype, i.e. RAFS (Robot-Assisted Fracture Surgery) system.

We propose a robot-bone attachment device for percutaneous bone manipulation, which can be anchored to the bone fragment through one small incision, ensuring the required stability and reducing the "biological cost" of the procedure. The device has been evaluated through the reduction of 9 distal femur fractures on human cadavers using the RAFS system.
\end{abstract}

\section{Introduction}

The treatment of lower limb fractures, including joint fractures, is a common task in orthopaedic surgery causing considerable health costs and patient disabilities (Mathew 2009). The standard procedure can be summarized in two steps: 1) fracture reduction, and 2) bone fragments fixation with intramedullary nails or plates and screws. A crucial step is the reduction which involves manipulating bone fragments to reconstruct the fracture as precisely as possible. Percutaneous techniques have been developed to mitigate the problems related to open surgery (i.e. extensive soft tissue damage, slow 
bone healing, increased risk of infection (Marsh 2015)), involving fragment manipulation using two (or more) pins inserted in the fragments through small incisions in the patient's flesh. Such techniques are associated with a faster recovery and a lower risk of infection compared to open surgical techniques (Gaston 2005). However, their application to joint fractures is limited by the current sub-optimal 2D intra-operative imaging (fluoroscopy) and by the high forces involved in the procedure. Integration of robotic assistance and 3D image guidance can increase reduction accuracy while using the minimally invasive access to the fracture fragments. Earlier research by the authors of this paper toward improving percutaneous reduction of intra-articular fractures has resulted in the creation of a robotic system prototype, i.e. RAFS (Robot-Assisted Fracture Surgery) system (Dagnino 2016a). This is an image-based robotic system which allows the surgeon to intraoperatively pre-plan the reduction of distal femur fractures (DFF), by virtually manipulating 3D models of the fracture generated by pre-operative CT data.

We here propose a robot-bone attachment device for percutaneous robot-assisted bone manipulation, which can be safely anchored to the bone fragment through one incision and with only one drilling of the bone, ensuring the required stability and reducing the "biological cost" of the procedure.

\section{Materials and Methods}

The attachment device was designed to be initially used in procedures for the reduction of distal femur fractures with two big fragments (e.g. 33-C1) using the RAFS system. It was designed to securely connect the robotic fracture manipulator (RFM) end-effector of the RAFS system and the bone fragment. It consists of the Unique Geometry manipulation Pin (UGP), the Anchoring System (AS), and the Gripping System (GS) (Fig.1). Clinical requirements were established through discussions with orthopaedic surgeons, analysis of various fracture cases (Dagnino 2015), and in-vivo measured forces applied by the surgeons during lower limb surgical procedures (Georgilas 2015). The UGP (Fig.1a) is a custom-designed orthopedic manipulation pin $(6 \mathrm{~mm}$ diameter (D), $142 \mathrm{~mm}$ length (L)). It has 4 parts: (i) gripping section (cylinder, $\mathrm{D}=4 \mathrm{~mm}, \mathrm{~L}=12 \mathrm{~mm}$ ) to be connected to the RFM end-effector; (ii) tool section $(\mathrm{L}=33 \mathrm{~mm})$, a three-flat-faces unique geometry to which a tool (e.g. optical tool for real time tracking) can be mounted in a unique orientation, enabling the 3D imaging system (Dagnino 2016b, 2016a); (iii) anchoring system section (L=67mm), a two-flat-faces geometry on which the AS is fixed. This geometry prevents the AS to rotate around the UGP; (iv) threaded section $(\mathrm{L}=30 \mathrm{~mm})$, a M6x1 metrical thread screwed into the bone fragment by surgeon. The AS (Fig.1b) is a custom designed system that firmly embeds the UGP into the bone fragment using a drilling template (DT) to hold four stainless steel nails. The surgeon drills a UGP into the bone fragment, slides the DT over and drills the 4 nails into the bone fragment through the holes on the DT. The AS assures that the UGP is securely connected to the bone fragment, avoiding any misalignment (especially rotations around the UGP longitudinal axis). The GS (Fig.1c) is mounted on the RFM endeffector and consists of an adjustable spherical joint that can freely orient a specially designed insert which fits the gripping section of the UGP and locks it with 4 grab screws. This configuration ensures that the force/torque applied by the RFM is fully transferred to the bone fragment to achieve the desired anatomical reduction.

The performance of the attachment device has been evaluated through the reduction of DFFs on 9 human cadaveric specimens using the RAFS system. The metric chosen for the assessment was the UGP-RFM connection stability defined as the relative pose displacement between the UGP and the RFM end-effector (Fig.1c). This was measured by an optical tracker by placing one optical tool on the tool section of the UGP and one optical tool on the RFM end effector. We have then tested the UGP through Finite Element Analysis (FEA) to understand its behavior under a predefined applied load 


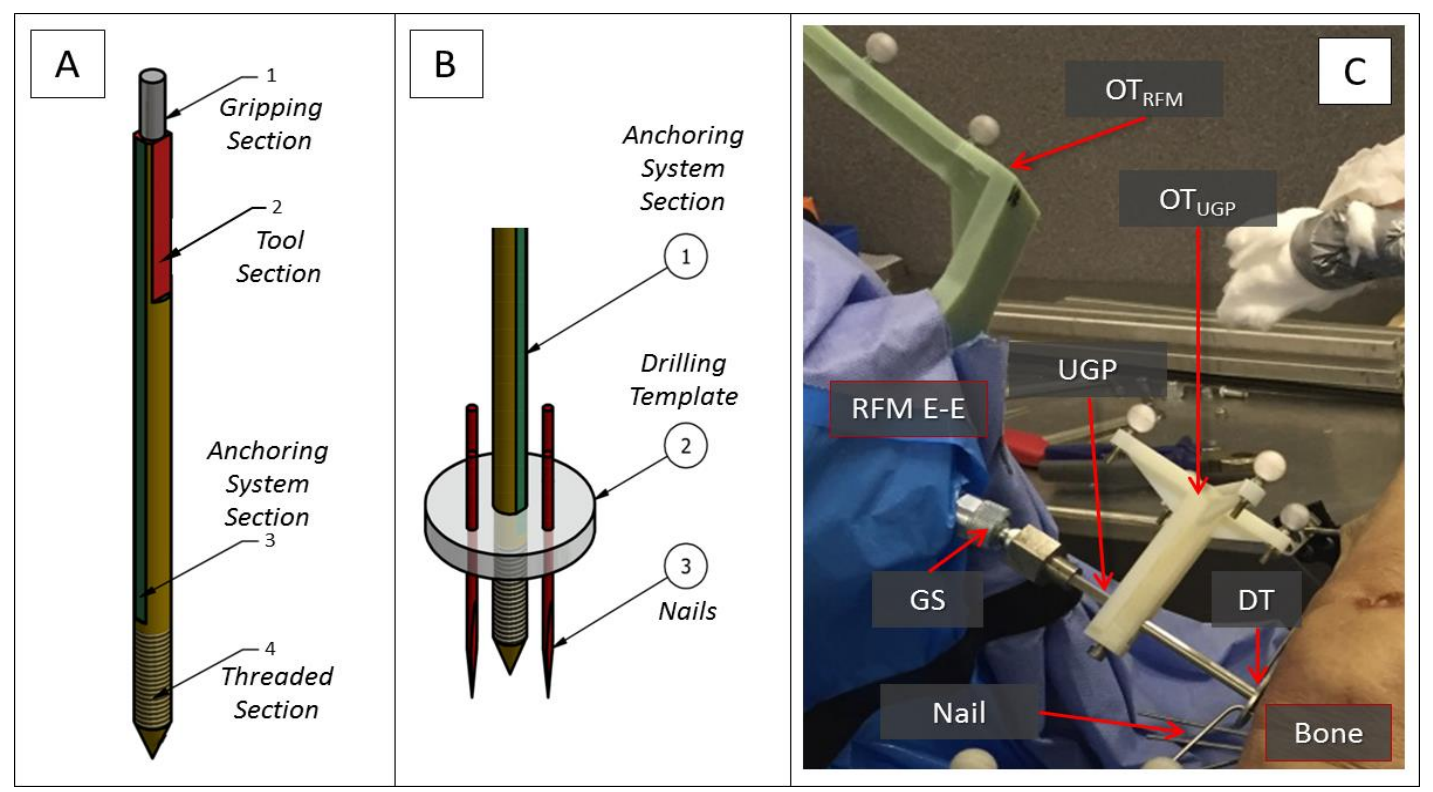

Figure 1: Robot-Bone Fixation System. CAD drawings of the Unique Geometry Pin (UGP) (A) and the anchoring system (AS) (B). The UGP is secured in the Gripping System (GS) and interconnects the RFM endeffector with the bone fragment. Optical tools are placed on the UGP $\left(\mathrm{OT}_{\mathrm{UGP}}\right)$ and the RFM $\left(\mathrm{OT}_{\mathrm{RFM}}\right)$ allowing the measurement of their relative pose $(\mathrm{C})$.

and to figure out how its bending can affect the physical reduction of the fracture. The metric chosen for this evaluation was the displacement (DISP) of the UGP under load. The main aim of this investigation was to determine the theoretical displacement of the UGP for forces/torque values applied by the RFMs in cadaveric trials. Data from this experiment, together with data from cadaveric trials, provide an estimation of the UGP bending influence on the fracture reduction. 3D models of the UGP and AS were created using Autodesk Inventor 2015. FEA was carried out in Autodesk Simulation Mechanical 2015. UGP and AP were assumed to be Stainless Steel 316, and their mechanical properties were set accordingly in the software. The threaded section of the UGP and the 4 nails of the AS were constrained as they are inserted into the bone. The FEA involved the combined application of linear (force) and rotational (torque) loads to the gripping section of the UGP, to simulate the load applied by the RFMs. We chose two different loads based on the data gathered in the cadaveric trials (see Table 1): (1) average load $\left(\mathrm{F}_{\mathrm{ave}}=75.7 \mathrm{~N}, \mathrm{~T}_{\mathrm{ave}}=3.5 \mathrm{~N}\right)$, and (2) maximum load $\left(F_{\max }=147 \mathrm{~N}, \mathrm{~T}_{\max }=6.8 \mathrm{Nm}\right)$. The corresponding displacements of the UGP have been calculated for the gripping and anchoring system sections through the FEA simulation (Table 2).

\section{Results}

Table 1 summarizes results from the cadaveric trials reporting the relative displacement between the UGP and the RFM end-effector with regard to the average load applied by the robot to reduce the fracture (measured by a 6-DOF load cell mounted on the RFM). Table 2 reports the results from the FEA simulation. 


\begin{tabular}{|c|c|c|c|c|c|c|c|c|c|}
\hline Specimen & $\# 1$ & \#2 & $\# 3$ & \#4 & $\# 5$ & \#6 & \#7 & $\# 8$ & \#9 \\
\hline RMD $^{\text {a }}$ & $\begin{array}{l}2.70 \mathrm{~mm} \\
3.10^{\circ}\end{array}$ & $\begin{array}{l}1.37 \mathrm{~mm} \\
1.60^{\circ}\end{array}$ & $\begin{array}{l}2.10 \mathrm{~mm} \\
1.80^{\circ}\end{array}$ & $\begin{array}{l}2.3 \mathrm{~mm} \\
3.35^{\circ}\end{array}$ & $\begin{array}{l}5.99 \mathrm{~mm} \\
5.52^{\circ}\end{array}$ & $\begin{array}{l}2.20 \mathrm{~mm} \\
3.07^{\circ}\end{array}$ & $\begin{array}{l}2.79 \mathrm{~mm} \\
2.29^{\circ}\end{array}$ & $\begin{array}{l}2.84 \mathrm{~mm} \\
18.1^{\circ}\end{array}$ & $\begin{array}{l}1.85 \mathrm{~mm} \\
2.48^{\circ}\end{array}$ \\
\hline $\begin{array}{l}F(\mathbf{N})^{b} \\
T(N m)^{b}\end{array}$ & $\begin{array}{l}69.9 \pm 4.4 \\
4.8 \pm 0.4\end{array}$ & $\begin{array}{l}113.1 \pm 5 \\
43.2 \pm 0.3\end{array}$ & $\begin{array}{l}18.0 \pm 0.5 \\
1.6 \pm 0.1\end{array}$ & $\begin{array}{l}94.6 \pm 5.1 \\
6.8 \pm 0.6\end{array}$ & $\begin{array}{l}147 \pm 10 \\
6.3 \pm 0.2\end{array}$ & $\begin{array}{l}82.7 \pm 7.5 \\
1.9 \pm 0.3\end{array}$ & $\begin{array}{l}25.9 \pm 7.4 \\
3.2 \pm 0.6\end{array}$ & $\begin{array}{l}56 \pm 11.9 \\
1.9 \pm 0.4\end{array}$ & $\begin{array}{l}74.5 \pm 8.1 \\
2.1 \pm 0.5\end{array}$ \\
\hline $\begin{array}{l}\text { Reduction } \\
\text { Accuracy }\end{array}$ & $A$ & $A$ & $A$ & A & A & $A$ & $A$ & $N A$ & $N A$ \\
\hline
\end{tabular}

Table 1: Results from cadaveric trials. ${ }^{a} \mathrm{RMD}=$ resultant maximum displacement (translational and rotational) between the UGP and the RFM end-effector. ${ }^{\mathrm{b}}$ Resultant average forces (F) and torques (T) applied by the RFM during the surgical procedure. ${ }^{\mathrm{c}} \mathrm{A}=$ acceptable, NA=not acceptable.

\begin{tabular}{|c|c|c|}
\hline $\begin{array}{c}\text { Load } \\
\text { Applied }\end{array}$ & $\begin{array}{c}\text { Gripper Section } \\
\text { (DISP) }\end{array}$ & $\begin{array}{c}\text { Unique } \\
\text { Geometry Section } \\
\text { (DISP) }\end{array}$ \\
\hline$F_{\text {ave }}=75.7 \mathrm{~N}$ & $2.8 \mathrm{~mm}$ & $0.19 \mathrm{~mm}$ \\
$T_{\text {ave }}=3.5 \mathrm{Nm}$ & & \\
\hline$F_{\max }=147 \mathrm{~N}$ & $5.89 \mathrm{~mm}$ & $0.52 \mathrm{~mm}$ \\
$T_{\max }=6.8 \mathrm{Nm}$ & & \\
\hline
\end{tabular}

Table 2: Results from FEA simulation.

\section{Discussion}

The proposed bone-robot attachment device allowed the RAFS system to reduce DFFs in 7 specimens (\#1 to $\# 7$ ) with clinical acceptable accuracy $\left(\leq 1 \mathrm{~mm}, \leq 5^{\circ}\right)$. The relative displacement between UGP and RFM, due to soft tissues-related forces and torques, was measured during each reduction. Whilst rotational RMD was used as a metric to evaluate safety of the connection between UGP and GS (i.e. RFM), linear RMD describes the bending of the UGP at the gripping section. Average linear and rotational RMD of $2.7 \mathrm{~mm}$ (maximum $5.99 \mathrm{~mm}$ ) and rotational $4.6^{\circ}$ (maximum $18.1^{\circ}$ ), respectively, were measured during cadaveric trials. The control system was able to compensate the RMD achieving clinically acceptable reduction accuracy (Marsh 2015) in specimens \#1 to \#7, also demonstrating that the RAFS system has a higher reduction accuracy when compared with other systems for fracture surgery reported in literature, e.g. (Tang 2012), (Graham 2006), (Wang 2013). The RAFS system was not able to reduce DFFs in specimens \#8 and \#9. Regarding specimen \#8, the GS failed and was not able to keep the UGPs stationary inside the RFM (UGP rotates inside the GS). In specimen \#9 the fracture was too dislocated, i.e. beyond the operational workspace capability of the robot.

The linear RMDs of $2.7 \mathrm{~mm}$ (average) and $5.99 \mathrm{~mm}$ (maximum, specimen \#5) measured during cadaveric trials (Table 1) were obtained for applied loads of $76 \mathrm{~N} / 3.5 \mathrm{~N}$ (average) and $147 \mathrm{~N} / 6.31 \mathrm{Nm}$ (maximum, specimen \#5). FEA (Table 2) of the UGP using the same load values, showed similar linear displacements for the gripping section, i.e. $2.8 \mathrm{~mm}$ (average load) and $5.89 \mathrm{~mm}$ (maximum load), proving the accuracy of the simulation. Moreover, the FEA simulation estimated the linear displacement of the unique geometry section of the UGP i.e. $0.19 \mathrm{~mm}$ (average load) and $0.52 \mathrm{~mm}$ (maximum load). The control system cannot compensate this displacement as it can't be estimated in real-time during the surgical procedure (a further optical tool on the threaded section would be needed), thus affecting the physical reduction accuracy of the fracture. 
In the future, a mathematical model of the UGP's force-displacement relation can be created based on further FEA simulations and confirmed through in-vivo trials, as muscular tension in live models might differ from that in cadaveric specimens. This model can be included in the RAFS control system allowing for the real-time estimation and compensation of the UGP displacement (including the gripping section) based on force/torque feedback provided by the load cells mounted on the RFM. Also, a more stable GS will be designed to avoid displacements between the UGP and the RFM.

\section{References}

Dagnino, G., Georgilas, I., Köhler, P., Atkins, R. and Dogramadzi, S. (2016a) Image-based robotic system for enhanced minimally invasive intra-articular fracture surgeries. In: 2016 IEEE International Conference on Robotics and Automation (ICRA). May 2016 pp. 696-701. doi:10.1109/ICRA.2016.7487196.

Dagnino, G., Georgilas, I., Köhler, P., Morad, S., Atkins, R. and Dogramadzi, S. (2016b) Navigation system for robot-assisted intra-articular lower-limb fracture surgery. International Journal of Computer Assisted Radiology and Surgery. doi:10.1007/s11548-016-1418-z.

Dagnino, G., Georgilas, I., Tarassoli, P., Atkins, R. and Dogramadzi, S. (2015) Vision-based realtime position control of a semi-automated system for robot-assisted joint fracture surgery. International Journal of Computer Assisted Radiology and Surgery. pp. 1-19. doi:10.1007/s11548015-1296-9.

Gaston P, Will EM and Keating JF (2005) Recovery of knee function following fracture of the tibial plateau. 87 (9), pp. 1233-1236.

Georgilas, I., Dagnino, G., Tarassoli, P., Atkins, R. and Dogramadzi, S. (2015) Preliminary Analysis of Force-Torque Measurements for Robot-Assisted Fracture Surgery. In: EMBC 2015 Conference. 2015 Milan, Italy: . doi:10.1109/EMBC.2015.7319491.

Graham, A.E., Xie, S.Q., Aw, K.C., Xu, W.L. and Mukherjee, S. (2006) Design of a Parallel Long Bone Fracture Reduction Robot with Planning Treatment Tool. In: 2006 IEEE/RSJ International Conference on Intelligent Robots and Systems. October 2006 pp. 1255-1260. doi:10.1109/IROS.2006.281885.

Marsh JL (2015) Rockwood And Green's Fractures In Adults. 8th edition. (no place) Wolters Kluwer.

Mathew, G. and Hanson, B.P. (2009) Global burden of trauma: Need for effective fracture therapies. Indian Journal of Orthopaedics. 43 (2), pp. 111-116. doi:10.4103/0019-5413.50843.

Tang P, Hu L, Du H, Gong M and Zhang L (2012) Novel 3D hexapod computer-assisted orthopaedic surgery system for closed diaphyseal fracture reduction. 8 (1), pp. 17-24. doi:10.1002/rcs.417.

Wang J, Han W and Lin H (2013) Femoral fracture reduction with a parallel manipulator robot on a traction table. doi:10.1002/rcs.1550.

\section{Disclosures}

This work is a summary of independent research funded by the National Institute for Health Research (NIHR)'s Invention for Innovation (i4i) Programme. The views expressed are those of the authors and not necessarily those of the NHS, the NIHR or the Department of Health. 